EUROPEAN ORGANIZATION FOR NUCLEAR RESEARCH

CERN-PPE/95-007

18.1.1995

\title{
TILTED FOIL POLARIZATION OF RADIOACTIVE IONS
}

\author{
M. Lindroos ${ }^{1}$, C. Broude ${ }^{2}$, G. Goldring ${ }^{2}$, H. Haas $^{3}$, M. Hass ${ }^{2}$, P. Richards ${ }^{4}$, L. Weissman ${ }^{2}$ \\ 1. ISOLDE, PPE Division, CERN CH-1211, Genéve, Switzerland \\ 2. Department of Particle Physics, Weizmann Institute of Science, Rehovot, Israel. \\ 3. Bereich F, Hahn-Meitner Institute, Berlin, Germany. \\ 4. Clarendon Laboratory, Oxford University, England, U.K. \\ and the ISOLDE Collaboration, CERN
}

\begin{abstract}
The tilted foil polarization technique has been applied to a beam of radioactive ${ }^{23} \mathrm{Mg}\left(I=3 / 2, T_{1 / 2}=\right.$ 11.35s) ions from ISOLDE. To achieve sufficient energy for penetration of the foils a high voltage platform has been constructed and used for acceleration of the initial $120 \mathrm{keV}$ beam of $2^{+}{ }^{23} \mathrm{Mg}$ ions to $480 \mathrm{keV}$. The resulting asymmetry $\epsilon$ of the emitted $\beta$-radiation from the ${ }^{23} \mathrm{Mg}$ implanted into a cold Pt stopper has been measured as a function of a holding magnetic field. At $B=0.09 \mathrm{~T}$ a value of $\epsilon=-7.4(1) 10^{-3}$ was found. The asymmetry was reduced to $\epsilon=-3.3(4) 10^{-3}$ at a field below $0.005 \mathrm{~T}$. It is argued that this hard core asymmetry occurs as a result of the internal interaction between the polarized nuclei and the defects created in the implantation process.
\end{abstract}




\section{Introduction}

Polarized nuclei are very useful for studies in a wide variety of fields ranging from solid state through nuclear to particle physics and several methods are available to obtain polarization for specific applications. For the polarization of radioactive nuclei, in particular, the methods of low temperature orientation and laserbeam optical pumping have been used very successfully. Both, however, can be applied in only a few special cases due to limitations in implant-host combinations and due to considerations of life-time/relaxationtime. With the ready availability of beams of shortlived radioactive nuclei at on-line isotope-separated, in particular the ISOLDE facility at CERN, a general technique to polarize nuclear beams could find many applications. The tilted foil polarization process appears to be such a universal method that extends over the entire periodic table. Upon exit from a thin foil at an angle to the surface of the foil the outgoing atoms are known to exhibit considerable electronic polarization ${ }^{1)}$ that can be transferred to the nuclei in flight through vacuum. In a multi-foil assembly ${ }^{2)}$, the induced nuclear polarization increases considerably, in particular for higher nuclear spin. Up to now this polarization method has been applied mainly to high spin isomers in measurements of signs of quadrupole moments ${ }^{3,4}$ ) and parity violation in a bound nuclear level ${ }^{5)}$. In a pioneering experiment $\left.{ }^{6}\right)$ at a recoil separator the $\mathrm{g}$-factor of the ground state of ${ }^{33} \mathrm{Cl}\left(I=\frac{3}{2}\right)$ was measured by applying the $\beta$-NMR technique to a beam of tilted-foil polarized nuclei. The observed polarization was rather low, lower than that normally attained by more conventional methods, (e.g., low temperature nuclear orientation or optical pumping). However, due to the universality of the TF process and the short time required for the polarization (of the order of nanoseconds), successful experiments can be performed where other methods fail.

In principle the degree of nuclear polarization by the Tilted-Foil (TF) method should be higher at low ion velocities, since the atomic polarization - averaged over all states of ionization and excitation - is known to exhibit this trend. Since the Booster-ISOLDE facility at CERN produces high quality slow radioactive ion beams for a wide range of elements, such a universal polarization technique can be well implemented. The aim of the tilted foil project at ISOLDE is to demonstrate this possibility and to extend the nuclear moment measurements to mirror nuclei, e.g., $T=\frac{3}{2}$ pairs in the sd shell and $T=\frac{1}{2}$ pairs in $f$ shell nuclei. We report here on the first steps towards achieving this goal by means of a new experimental setup: the ISOLDE beam is boosted in energy by a $260 \mathrm{kV}$-maximum high voltage platform on which the whole experiment is carried out. The nucleus ${ }^{23} \mathrm{Mg}\left(I=\frac{3}{2}\right)$ has been chosen as the first test case; the $g$-factor of this state has recently been measured by the nuclear-reaction polarization method ${ }^{7}$. In the course of our measurements we have observed a for the ${ }^{23} \mathrm{Mg}$ nuclei implanted into a Pt matrix a dependence of the nuclear polarization on the applied holding field. The demonstration of nuclear polarization of an isotope-separated beam and 
the analysis of the decoupling effects are the principal subjects of this communication.

\section{The polarization process}

The atomic polarization created by passage of an ion beam through a foil is understood qualitatively in terms of pickup of electrons with polarized orbital angular momentum upon exit from the foil. It is a strong function of the tilt angle, being obviously zero at normal incidence and reaching maximal values at very large angles. It is basically a similar mechanism to that responsible for the atomic polarization in the grazing angle geometry $^{8)}$. The transfer of this atomic polarization to the nucleus on subsequent flight through vacuum may be calculated if the atomic states and their hyperfine interaction constants are known. On passage through each foil the atomic polarization is restored, leading to a build-up of nuclear polarization in the following free flight. This multi-foil nuclear polarization process and its formalism have been described in detail in several papers e.g., Goldring and Niv ${ }^{9}$ ). One of the main results of these studies is that the nuclear

polarization $p_{I}=\frac{\left\langle I_{z}\right\rangle}{I}$ may be described to a good approximation by a general formula, valid also for low spins where quantum effects are important:

$$
p_{I}(N) \sim \frac{I+1}{J+1}\left\{1-Q^{N}\right\} p_{J}
$$

Here $p_{J}$ is the atomic polarization of the relevant ions at the exit surface of the foils, $N$ is the number of foils and $Q$ is given approximately for small $p_{J}$ by:

$$
\begin{array}{ll}
I / J>1 & 1-\frac{2}{3} \frac{J^{2}}{I^{2}} \\
I / J=1 & \frac{1}{2} \\
I / J<1 & \frac{1}{3}
\end{array}
$$

These formulae were developed for the high atomic excitation regime encountered at relatively high ion velocities. In the present experiment the ion velocity is rather low: $v \sim 0.007 \mathrm{c}$; but following the results and reasoning of refs. 10,11, one would expect also in this case a rich mixture of excited states, nearly all with the ground state principal quantum number, $n=3$. Consequently the approximate formulae above are expected to be valid also in this case.

\section{The $\beta$ angular distribution}

The angular distribution of radiation emitted from an axially symmetric ensemble of oriented nuclei is given by:

$$
W(\theta)=\sum_{\lambda} Q_{\lambda} B_{\lambda} A_{\lambda} P_{\lambda}(\cos \theta)
$$


where $B_{\lambda}$ are the orientation parameters and $Q_{\lambda}$ are the finite-angle attenuation coefficients. The angle $\theta$ is measured from the direction of polarization. For parity conserving interactions only even terms in $\lambda$ are non-zero but for electrons from $\beta$-decay the leading term in (1) is

$$
W(\theta)=1+Q_{1} B_{1} A_{1} \cos \theta
$$

were

$$
B_{1}=\sqrt{\frac{3 I}{(I+1)}} \frac{<I_{z}>}{I}
$$

and

$$
A_{1}=\frac{v}{c} \sqrt{\frac{1}{3 I(I+1)}} \cdot \frac{\mp 1+2 y \sqrt{I(I+1)}}{1+y^{2}}
$$

were $\mp$ corresponds to $\beta^{+}$- and $\beta^{-}$- decay respectively. $y$ relates to the Fermi/Gamow-Teller mixing and is defined as:

$$
y=\frac{C_{V}<1>}{C_{A}<\sigma>}
$$

using the notation of V-A interaction theory for $\beta$-decay. In our geometry $Q_{1}$ is close to unity.

The $\beta$-decay of ${ }^{23} \mathrm{Mg}$ is well understood, and an anisotropy of $A_{1}=0.42(v / c)$ is calculated, independent to a large extent of the exact value of the Fermi/Gamow Teller mixing. To measure the nuclear polarization, the beta radiation is typically observed in two detectors placed parallel and antiparallel to the polarization direction. In a measurement with radioactive nuclei generally an external "holding field" $B_{e x t}$ is applied parallel to the direction of $p_{I}$, to produce Zeeman splitting and so preserve the induced polarization. The anisotropy $\epsilon$ is defined as:

$$
\epsilon=\frac{W\left(0^{\circ}\right)-W\left(180^{\circ}\right)}{W\left(0^{\circ}\right)+W\left(180^{\circ}\right)}
$$

In order to avoid systematic errors associated with the efficiences and dead times of the detectors as well as trajectories of the electrons/positrons in the holding field, one defines a double-ratio $\rho$ :

$$
\rho=\left[\frac{N(L) \leftarrow}{N(R) \leftarrow} \times \frac{N(R) \rightarrow}{N(L) \rightarrow}\right]^{1 / 2}
$$

where $N(L) \leftarrow$ corresponds, for example, to counts in the detector to the left of the beam with the polarization in the "left" direction. In the tilted-foil polarization experiment, "left" and "right" polarization is switched periodically by rotating the foil stack by $180^{\circ}$. For small $\epsilon(\rho \approx 1)$, the asymmetry is given by:

$$
\epsilon=\frac{\rho-1}{\rho+1}
$$

In an NMR experiment with polarized radioactive nuclei the total or partial destruction of the anisotropy of the emitted radiation at the resonant frequency: $\nu=g \mu_{n} B_{e x t}$ is measured ${ }^{12)}$. 


\section{Experimental set-up and procedure}

The acceleration voltage used at the ISOLDE installation is $60 \mathrm{kV}$, thus providing singly charged ions of $60 \mathrm{keV}$. This energy is not sufficient to traverse even the thinnest polarizing foils. Since it is technically not possible to significantly increase the high voltage of the ion source, an additional acceleration had to be provided in our experiment. This has been achieved by means of a high voltage platform on which the experiment is mounted and which can at present be operated at negative voltages up to $260 \mathrm{kV}$. The set-up is shown in fig. 1. The main external components are a grounded outer cage, an acceleration tube and the inner HV cage. The total maximum acceleration voltage experienced by the ions at the experiment (on the HV platform) is thus $320 \mathrm{kV}$, corresponding to an energy of $640 \mathrm{keV}$ for $2^{+}$ions. For the first experiment, a safe voltage of $-180 \mathrm{kV}$ was used, corresponding to ${ }^{23} \mathrm{Mg}$ at $480 \mathrm{keV}$. Situated within the HV cage is the foil stack and a liquid-helium cryostat housing a superconducting coil which provides a holding field of up to $0.25 \mathrm{~T}$. The cryostat is also used to cool the beam stopper to a low temperature of about $5 \mathrm{~K}$, necessary for preserving the polarization of the implanted ions for sufficiently long times. The foil stack can be rotated between two positions, "right" and "left", yielding opposite polarization in the horizontal field direction. The stack can also be retracted beyond an isolating valve which allows the vacuum in the cryostat to be maintained while the foils are handled in air. The positrons from the decay of ${ }^{23} \mathrm{Mg}$ emerge from the cryostat through thin aluminium windows and are detected in two plastic scintillators. Communication with the platform, in particular the transmission of the data from the data acquisition system on the HV platform, is achieved via an optical fibre ETHERNET link. Electrical power for the equipment on the HV platform is supplied by a motor generator driven by a rotating shaft. Fig. 2 is a photograph of the experimental setup.

\section{The ${ }^{23} \mathrm{Mg}$ experiment}

The ${ }^{23} \mathrm{Mg}$ activity was produced in the spallation reaction of $1.0 \mathrm{GeV}$ protons on a $\mathrm{SiC}$ target and ionized in a heated plasma ion source at the ISOLDE General Purpose Separator. At a proton beam of 1 $\mu \mathrm{A}$ we obtained $2.9 \times 10^{7}{ }^{23} \mathrm{Mg}^{+}$ions per second. The $2^{+}$charge state yield was a factor of ten lower and the transmission into the beam stopper was roughly $5 \%$, resulting in a final yield of approximately $5 \times 10^{4}$ ${ }^{23} \mathrm{Mg}^{++}$ions per second passing through the polarizing foils. This poor transmission will be improved in future experiments.

Asymmetry measurements were carried out as a function of the holding field $B_{\text {ext }}$ for $2^{+}$ions of ${ }^{23} \mathrm{Mg}$ $\left(T_{1 / 2}=11.3 s, I=3 / 2\right)$ with an energy of $480 \mathrm{keV}$, stopping in platinum at a temperature of $\sim 5 K$. Two foils were used in most of the measurements, but two check runs with respectively one and zero foils were also performed. The results are presented in table I and fig. 3. At the energy of the experiment the predominant charge states of the $\mathrm{Mg}$ ions after passage through a foil ${ }^{13)}$ are $0,1^{+}, 2^{+}$and hence the atomic 
configurations which exhibit polarization $(l \neq 0)$ are all in the $n=3$ major shell. This situation is similar to the regime described in refs. $(10,11)$ and the assumption of statistically populated configurations and terms within a particular major shell can be applied. The observed 2-foil/1-foil ratio for the asymmetry: $\frac{0.7410^{-3}}{0.5210^{-3}}=1.42$ agrees with estimates from eqn. (1) which, for $J$ in the range $1-3$, vary from 1.3 to 1.65. The "zero-foil" result yielded a null asymmetry as expected. The net polarization, $p_{I}$, for two foils is $1.5 \%$ where we have taken $\mathrm{v} / \mathrm{c}$ as 0.86 corresponding to the lower integration limit in the $\beta$ spectrum. For the purpose of illustration, for $I=J$ (and hence $Q=1 / 2$ in eqn. 2), this value of $p_{I}$ corresponds to $p_{J} \approx 0.02$. This estimate does not take into account possible relaxation losses in the Pt matrix and possible depolarization effects in the fringe fields of the super-conducting magnet. These effects will be investigated in future experiments.

\section{The Decoupling curve}

A nucleus in a solid environment is subject to static or dynamic interactions of magnetic dipolar or electric quadrupolar type. For sufficiently long life-times of the decaying nucleus, all of these can lead to destruction of the initial nuclear polarization. However, an external magnetic field ("holding field") of sufficient strength applied along the polarization direction will decouple the nucleus from such fields and thus preserve the polarization for static interactions. At the low temperature of this experiment, dynamic effects other than spin-lattice interaction due to the conduction electrons can safely be neglected. An estimate of the lower limit for the spin-lattice relaxation time may be obtained by the use of the Korringa relation. One can safely assume that the spin-lattice relaxation due to the contact term for $\mathrm{Mg}$ in $\mathrm{Pt}$ is not faster than for $\mathrm{Mg}$ in Ag. For this latter system the comparison of Knight shift data of Cd impurities, isovalent with Mg, in Ag after proper scaling for the nuclear g-factors with Knight shifts of $\mathrm{Cd}$ and $\mathrm{Mg}$ leads to $T_{1} T=70 \mathrm{~s} K$ for ${ }^{23} \mathrm{Mg}$ in $\mathrm{Ag}$ (see, e.g., ref. 14). For a temperature of $5 \mathrm{~K}$ this yields a lower limit for the spin-lattice relaxation time in the present system of $14 \mathrm{~s}$, longer than the nuclear half-life. In the cubic matrix of Pt, only the dipolar fields (due to neighbouring nuclei) and the electric field gradients (due to lattice imperfections) can therefore contribute to the depolarization process. The dipolar fields, created by the ${ }^{195} \mathrm{Pt}$ isotope at a substitutional lattice site can be quantitatively calculated using the Van Vleck method. They should be decoupled $\left.{ }^{15}\right)$ by external fields of the order of $1 \mathrm{G}$, in contradiction to our experimental result. The dominant interaction is therefore presumably the electric field gradient (EFG) due to the defects created during implantation.

For a quantitative analysis of the decoupling curve measured in the present experiment one has to know the nuclear moments of ${ }^{23} \mathrm{Mg}$ and also the electric field gradient acting on the nucleus. For the nuclear moments one can resort to shell model calculations which are expected to be quite reliable for nuclei near closed shells, as well as to a recent measurement ${ }^{7)}$. The calculations yield the values $\mu=-0.428 \mu_{N}$ and 
$\mathrm{Q}=0.10 \mathrm{~b}^{16)}$ and the predicted magnetic moment is in quite good agreement with the experimental result.

Fortunately there exist experimental data which allow an estimate of the average EFG acting at the $\mathrm{Mg}$ nuclei site after implantation. The value of the EFG for the isoelectric system $\mathrm{Cd}$ in $\mathrm{Pd}$ after implantation is experimentally known ${ }^{17}$ ) to be $2.7 \mathrm{~V} / \AA^{2}$. The atoms $\mathrm{Mg}$ and $\mathrm{Cd}$ have almost the same metallic radius. The ratio of the field gradients measured in the hexagonal matrix $\mathrm{Mg}, \frac{q(M g M g)}{q(C d \underline{M g})}=0.12$, accounts for the different radial functions for the $3 \mathrm{p}$ and $5 \mathrm{p}$ valence electrons contributing to the $\mathrm{EFG}$ for $\mathrm{Mg}$ and $\mathrm{Cd}$, respectively. Neglecting the difference between $\mathrm{Pd}$ and $\mathrm{Pt}$, which are quite similar metals in their electronic structure, an estimate can thus be obtained as $E F G_{a v}(M g \underline{P t})=E F G_{a v}(C d \underline{P d}) \times \frac{q(M g \underline{M g})}{q(C d \overline{M g})}=0.32 V / \AA^{2}$. The resulting average nuclear quadrupole interaction frequency for ${ }^{23} \mathrm{Mg}$ in $\mathrm{Pt}$ is thus $\nu_{q}=0.08 \mathrm{M} \mathrm{Hz}$.

With these numbers the expected effect of the holding field on the observed beta-anisotropy, the decoupling curve, can in principle be calculated. Unfortunately there seems to be no calculation for a randomly oriented field gradient in the literature. One can estimate from the available calculations for single crystals, however, that a good approximation to a full calculation of the decoupling curve can be obtained by a Lorentzian distribution around zero field with halfwidth $B_{1 / 2}=0.5 h \nu_{q} / \mu_{N}$. For the parameters given above this yields a halfwidth of $0.02 \mathrm{~T}$. A Lorentzian function was fitted to the experimental data in Fig. 3 . It accounts well for the measurement. The similarity of the fitted width $(0.01 \mathrm{~T})$ to the calculated one strongly suggests that the width is indeed dominated by the quadrupole interaction induced by implantation defects.

The non-zero asymmetry "hard core" value found at zero external field can be qualitatively explained as follows. The initially polarized ensemble of nuclei will, in the absence of a sufficiently strong external field, precess in the internal randomly oriented electric field gradients resulting in a time-averaged none-zero asymmetry. 


\section{Future Prospects}

The development of the tilted foil nuclear polarization technique combined with beta-NMR opens various new possibilities for nuclear and solid state studies. Determination of the magnetic moment of ${ }^{21} \mathrm{Mg}$ and nuclei in the $f$ shell will be of much interest. Still to be completed is an accurate measurement of the nuclear quadrupole moment of ${ }^{23} \mathrm{Mg}$. For the study of solids this new technique offers a wide spectrum of new applications. The most interesting systems would probably be defects and impurities in semiconductors, at present very successfully studied albeit with only a few available beta-NMR probes.

\section{Acknowledgement}

We would like to thank Dr. N.J. Stone and Dr. J. Rikovska-Stone for providing the cryogenic equipment, and for their assistance in the early stages of this work. We also would like to acknowledge the technical staff at the Clarendon laboratory and at the Physics Workshop of the Weizmann Institute for technical assistance. M. Lindroos acknowledges the Swedish Institute and Prof. B. Jonson's group at Chalmers University of Technology for financial support. 
Table I

\begin{tabular}{|c|c|c|}
\hline Field (Tesla) & Asymmetry $\epsilon$ & Number of foils \\
\hline-0.09 & $-0.0005(4)$ & 0 \\
\hline-0.09 & $-0.0052(1)$ & 1 \\
\hline-0.09 & $-0.0078(4)$ & 2 \\
\hline 0.09 & $0.0074(1)$ & 2 \\
\hline 0.027 & $-0.0067(4)$ & 2 \\
\hline 0.009 & $-0.0064(4)$ & 2 \\
\hline 0.005 & $-0.0052(4)$ & 2 \\
\hline 0.003 & $-0.0029(4)$ & 2 \\
\hline 0.0 & $-0.0033(4)$ & 2 \\
\hline
\end{tabular}




\section{References}

1. H.G. Berry and M. Hass, Ann. Rev. Nucl. Part. Sci. 32, 1 (1982); and references therein.

2. M. Hass, E. Dafni, H.H. Bertschat, C. Broude, F. Davidovsky, G. Goldring and P.M.S. Lesser, Nucl. Phys. A414, 316 (1984).

3. E. Dafni, J. Bendahan, C. Broude, G. Goldring, M. Hass, M. H. Rafailovich, C. Chasmann, O.C. Kistner and S. Vajda, Phys. Rev. Lett. 56, 2473 (1984).

4. M. Hass, M. Satteson, C.Y. Wu, C. Broude and N. Benczer-koller, Phys. Rev. C43, 2140 (1991).

5. C. Broude, G. Goldring, M. Hass, N. Takahashi, S. Hofmann, F.P. Hessberger and V. Ninov, Z. Physik A336, 133 (1990).

6. W.F. Rogers, D.L. Clark, S.B. Dutta and A.G. Martin, Phys. Lett. B117, 293 (1987).

7. M. Fukuda, T. Izumikawa, T. Ohtsubo, M. Tamigaki, S. Fukuda, Y. Nakayama, K. Matsuta, Y. Nojiri and T. Minamisomo, Phys. Lett. B307, 278 (1993).

8. See e.g., H.J. Andrä, R. Fröhling, H.J. Plöhn GH, H. Winter and W. Wittmann, J. Phys. C1 275 (1979); and references therein.

9. G. Goldring and Y. Niv, Hyperf. Interact. 21, 209 (1985).

10. C. Broude, M.B. Goldberg, G. Goldring, M. Hass, M.J. Rennan, B. Sharon, Z. Shkedi and D.F.H. Start, Nucl. Phys. A215, 617 (1973).

11. H.C. Jain, A. Little, S.M. Lazarus, T.K. Taylor, B.B. Triplett and S.S. Hanna, Phys. Rev. C14, $2013(1976)$.

12. See, e.g., K. Krane, in Low-temperature nuclear orientation, eds. N.J. Stone and H. Polstma (North Holand, Amsterdam) 1986, p31; and references therein.

13. H.D. Betz, Rev. Mod. Phys. 44, 465 (1972).

14. G.C. Carter, L.H. Bennet and D.J. Kahan, Metallic shifts in NMR, Pergamon Press, Oxford, 1977.

15. A. Losche, Kerninduktion, VEB Deutscher Verlag der Wissenchaften, Berlin, 1957.

16. M. Carchidi, B.H. Wildenthal and B.A. Brown, Phys. Rev. C34, 2280 (1986).

17. H.H. Bertschat, O. Echt, H. Haas, E. Ivanov, F. Pleiter, E. Recknagel, E. Schlodder and B. Spellmeyer, Hyper. Interact. 2, 339 (1976). 


\section{Figure Captions}

Figure 1 The $60 \mathrm{keV}$ ISOLDE beam is boosted in energy by a $260 \mathrm{kV}$ high voltage platform on which the whole experiment is carried out. Using doubly charged ions a maximum beam energy of $640 \mathrm{keV}$ can be reached. The main elements of the platform are a grounded outer faraday cage, an acceleration tube and an inner high voltage cage. Situated within the HV cage is the foil stack and a liquid helium cryostat housing a superconducting magnet. Communication with the platform is maintained with an optical ETHERNET link. Electrical power for the equipment is provided by a motor generator. The detailed geometry of the beam, foils, superconducting and rf coils and the electron detectors are shown in the inset.

Figure 2 The high-voltage platform. The outer faraday cage and the inner HV cage are partly dismantled to exhibit the cryostat (A), the magnet housing (B), the motor generator (C), the plastic shaft (D), the acceleration tube (E), and the electron detectors (F).

Figure 3 The asymmetry of tilted-foil polarized ${ }^{23} \mathrm{Mg}$ implanted into a cold (approximately $5 \mathrm{~K}$ ) $\mathrm{Pt}$ stopper as a function of an external holding field parallel to the direction of polarization (see text). A good approximation to the presently observed decoupling effect can be obtained by a Lorenzian distribution around zero field with a halfwidth of $0.01 \mathrm{~T}$. Such a curve is drawn through the experimental data points (triangles). The measured asymmetries for one foil and zero foils are marked in the figure with circle and diamond respectively. 